\title{
DESIGN OF A MAGNET SYSTEM FOR A MUON COOLING RING EXPERIMENT
}

\author{
S. Kahn, Brookhaven National Laboratory, Upton, NY 11973 \\ G. Hanson and P. Schwandt, Indiana University, Bloomington, IN 47405 \\ V. Balbekov, R. Raja, and Z. Usubov, Fermilab, Batavia, IL 60510
}

\begin{abstract}
:
A muon cooling ring is currently being investigated as a possible muon cooling experiment that can demonstrate 6D cooling. The cooling ring consists of long sole-noid channels with liquid $\mathrm{H}_{2}$ absorbers and $\mathrm{RF}$ cavities, shorter field flipped solenoids with $\mathrm{LiH}$ wedge absorbers and wedge shaped $45^{\circ}$ bending dipole magnets. The ring is designed to cool muons with $\mathrm{E}_{\mathrm{kin}}=250 \mathrm{MeV}$. This study examines the magnetic system of the muon ring cooler. The purpose at this point is to establish physical fields for the magnetic system that can be used in tracking studies.
\end{abstract}

\section{INTRODUCTION}

This letter studies the magnet system for a muon cooling ring that is being investigated as a possible approach to muon cooling for a neutrino factory or muon collider [1]. This cooling ring would cool both in transverse and longitudinal phase space. Figure 1 shows a diagram of the muon cooling ring. This ring consists of four long solenoid channels containing RF cavities and a liquid $\mathrm{H}_{2}$ absorber. At each corner there is a shorter field flipping solenoid containing a wedge shaped $\mathrm{LiH}$ absorber. Two $45^{\circ}$ wedge shaped dipole bend magnets surround the short solenoids at each corner.

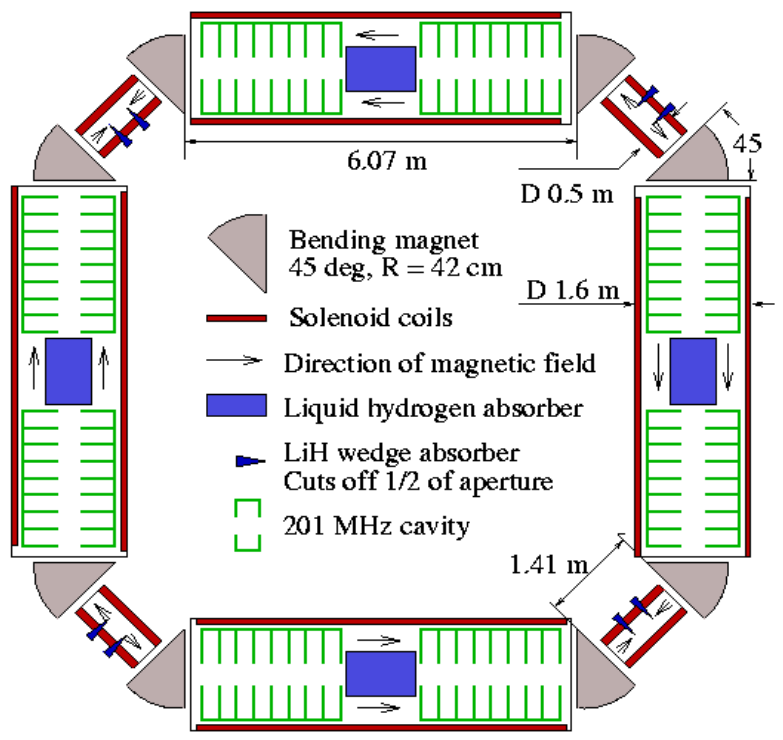

Figure 1: Diagram of muon cooling ring.
Table 1 summarizes the parameters that specify the magnet system for the cooling ring.

Table 1: Parameters describing the muon cooling ring magnet system.

\begin{tabular}{ccc}
\hline Parameter & Symbol & Value \\
\hline Muon Momentum & $\mathrm{P}_{\mu}$ & 226 \\
& & $\mathrm{MeV} / \mathrm{c}$ \\
Bend Radius & $\rho_{\mathrm{o}}$ & $52 \mathrm{~cm}$ \\
Bend Angle & $\theta_{\text {bend }}$ & $45^{\circ}$ \\
Dipole Field & $\mathrm{B}_{\mathrm{o}}$ & $1.45 \mathrm{tesla}$ \\
Focusing Index & $(\mathrm{R} / \mathrm{B}) \times\left(\mathrm{dB}_{\mathrm{y}} / \mathrm{dr}\right)$ & $-1 / 2$ \\
Radial Aperture & $\mathrm{R}_{\mathrm{aper}}$ & $17 \mathrm{~cm}$ \\
Long Solenoid Field & $\mathrm{B}_{\mathrm{ssol}}$ & $2.5-4$ tesla \\
Long Solenoid Half & $\mathrm{L}_{\mathrm{lsol}}$ & $3.2 \mathrm{~m}$ \\
Length & & \\
Field Flip Solenoid Field & $\mathrm{B}_{\mathrm{ssol}}$ & $3.5 \mathrm{tesla}$ \\
Short Solenoid Half & $\mathrm{L}_{\mathrm{ssol}}$ & $0.6 \mathrm{~m}$ \\
Length & & \\
RF Cavity Gradient & $\mathrm{E}_{\mathrm{rf}}$ & 15.5 \\
& & $\mathrm{MV} / \mathrm{m}$ \\
\hline
\end{tabular}

\section{DIPOLE MAGNET}

The dipole magnet must bend a muon beam with $15 \mathrm{~cm}$ radius and $\left\langle\mathrm{p}_{\mu}\right\rangle=226 \mathrm{MeV} / \mathrm{c}$ through an angle of $45^{\circ}$ while providing focusing in both the horizontal and vertical planes. The bend magnet must be compact in size and fit between high field solenoid magnets on each side. This combined function dipole magnet consists of a Cshaped iron yoke with (most likely) superconducting coils and field clamps to control the fringing field. The overall geometry of the ring requires that the bend radius of the magnet be small. The beam reference radius $r_{0}=52 \mathrm{~cm}$ is a compromise between ring requirements and magnet feasibility. Since the effective field width $\mathrm{W}_{\mathrm{eff}}=\theta_{\mathrm{o}} \mathrm{r}$ along the beam path is comparable to the pole gap $G$, this magnet will require 3D computer modeling of the field. In order to achieve equal focusing in both planes the field index should be $n=-\left(r / B_{y}\right)\left(d^{y} / d r\right)=1 / 2$. This gives the desired field profile of $B_{y}(r) \propto r^{-1 / 2}$. The pole gap radial profile is designed to achieve this field shape. Table 2 shows the pole half-gap as a function of the radial position. The effective field boundaries, whose separation along any beam path is given by $W_{e f f}(r)=\frac{1}{B_{y o}} \int r B_{y}(r, \theta) d \theta$, should lie along the radial lines $\theta= \pm 22.5^{\circ}$ with respect to the centerline of the 
magnet. In the absence of field clamps, the very large pole gap would cause the physical boundaries of the pole to lie well inside the effective field boundaries. Table 2 also shows the physical half width of the pole with field clamps as a function of the radial position. Figure 2 shows a radial cross section of the magnet illustrating the pole gap.

Table 2: Parameters that describe the pole design of the dipole magnet as a function of radial position. All values are in $\mathrm{cms}$.

\begin{tabular}{c|cc}
$\mathbf{R}$ & Half Gap & Half Width \\
\hline $\mathbf{3 0}$ & 5.0 & 11.5 \\
$\mathbf{3 7}$ & 8.0 & 13.5 \\
$\mathbf{4 4}$ & 11.3 & 15.3 \\
$\mathbf{5 2}$ & 15.4 & 17.3 \\
$\mathbf{6 0}$ & 21.1 & 22.3 \\
$\mathbf{6 7}$ & 23.9 & 25.4 \\
$\mathbf{7 5}$ & 25.7 & 29.0
\end{tabular}

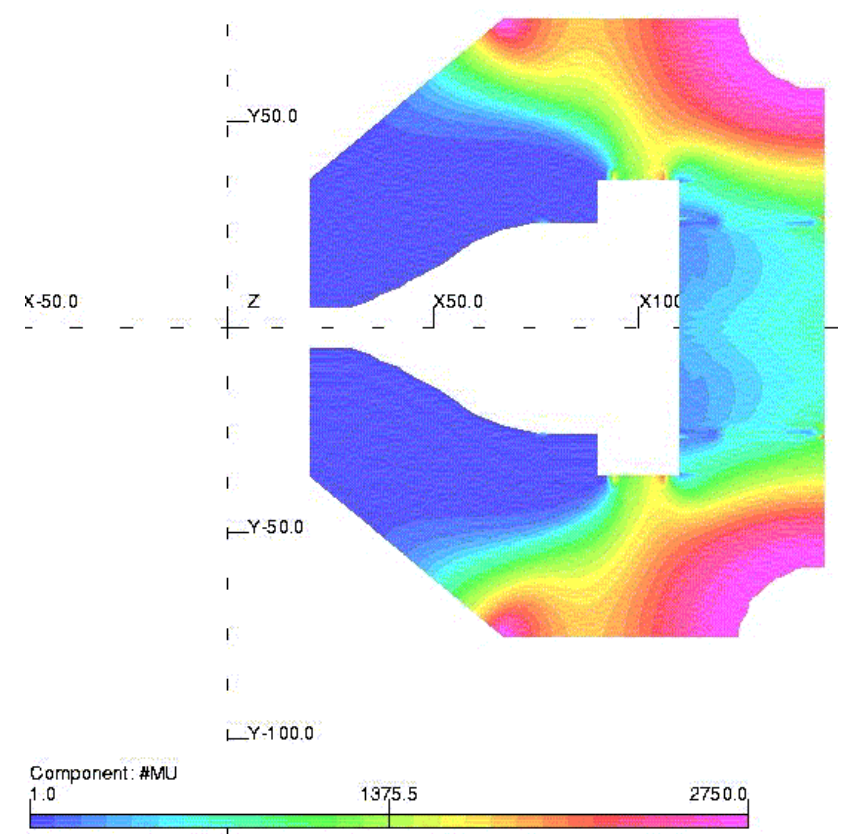

Figure 2: The drawing shows a cross section of the dipole magnet cut along the symmetry plane. A contour plot of the iron permeability is shown superimposed.

Figure 3 shows three curves which compare the $\mathrm{B}_{\mathrm{y}}$ field component as a function of the transverse radial position. One curve shows the 3D field calculation that was performed using the TOSCA electromagnetic finite element program [2]. A 2D cylindrically symmetric calculation using the same pole profile is also shown. It does not agree well with the 3D calculation because the magnet width is comparable with the pole gap. A better comparison is with the ideal $2 \mathrm{D}$ design which has a pole chosen to give the desired $B_{y}(r) \sim r^{1 / 2}$ behavior. Its agreement with the $3 \mathrm{D}$ calculation is reasonably good for the region occupied by the beam. The field index, $n$, can be determined by fitting a polynomial to the beam region, $37<\mathrm{r}<66 \mathrm{~cm}$. The index for the $3 \mathrm{D}$ model is $\mathrm{n}_{3 \mathrm{D}}=0.470$ to be compared to $\mathrm{n}_{2 \mathrm{D} \text { ideal }}=0.473$ for the ideal $2 \mathrm{D}$ model. As shown in figure 2 , the iron in the pole is quite saturated. It has permeability less than 10 on the pole surface.

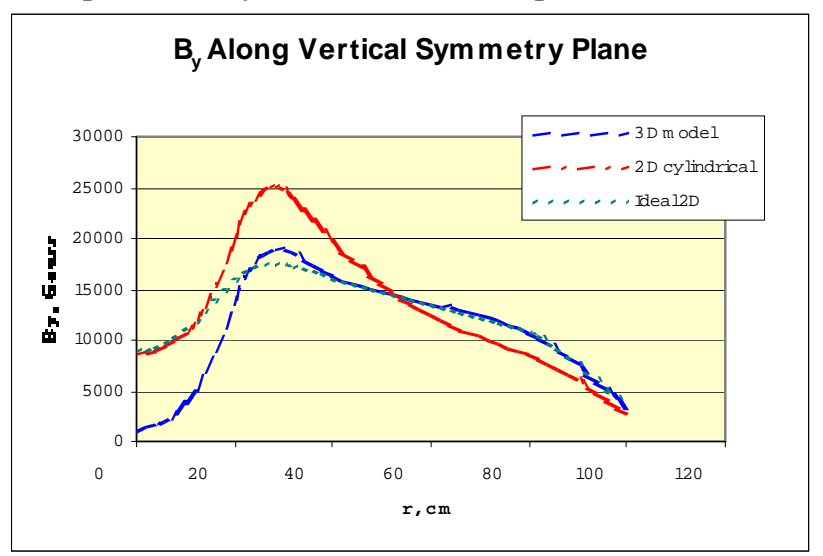

Figure $3: \mathrm{B}_{\mathrm{y}}$ is shown along mid-plane of symmetry plane of the dipole magnet. The blue line shows the curve from the $3 \mathrm{D}$ calculations. The red line shows the $2 \mathrm{D}$ cylindrically symmetric calculations with the same pole shape. The green line shows the ideal $2 \mathrm{D}$ case where the pole is shaped to produce the appropriate index.

The field along the center reference path through the dipole magnet is shown in figure 4. This path is an arc of radius $52 \mathrm{~cm}$ inside the dipole magnet and extends as a straight line outside the magnet. The graph shows $B_{y}$ on axis and $B_{x}, B_{y}$, and $B_{z} 10 \mathrm{~cm}$ above the reference path.

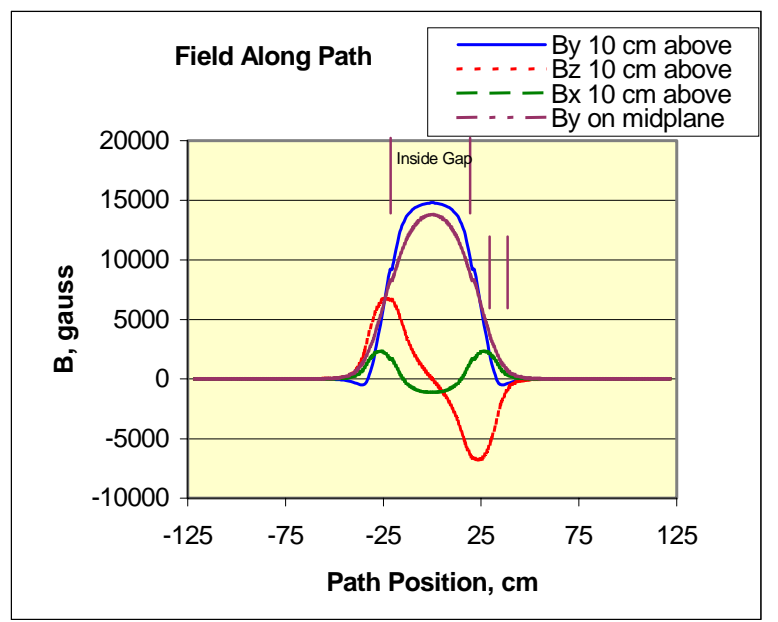

Figure 4: Field along reference path and $10 \mathrm{~cm}$ above.

\section{LONG SOLENOID CHANNEL}

Each of the four sides of the cooling ring consists of a 6.3 meter long solenoid channel containing RF cavities and a liquid $\mathrm{H}_{2}$ absorber in the center. The axial field in the solenoid varies between 2.5 and 4.5 tesla. The higher 4.5 tesla field is required at the absorber to be most effective. The solenoid channel consists of coils with the radius of $80 \mathrm{~cm}$ surrounded 
by an iron flux return. The current density in the coils around the absorber is increased to provide the higher flux required at that position. The cylindrical part of the flux return has a thickness of $33 \mathrm{~cm}$ to contain the returned flux. The end plate of the solenoid is highly saturated as expected. Several designs for the endplate of the solenoid have been investigated. The design used in these calculations consists of a $20 \mathrm{~cm}$ thick plate of vanadium steel $\left(M_{s}=2.4\right.$ tesla $)$ with a second $5 \mathrm{~cm}$ plate $(1010$ steel $)$ separated by a $5 \mathrm{~cm}$ gap.

Magnetic fields for the solenoid magnet were made using the Opera2D finite element program [3]. Figure 5 shows the axial component, $\mathrm{B}_{\mathrm{Z}}$, as a function of the axial position. The field is 4.3 tesla in the vicinity of the $\mathrm{H}_{2}$ absorber and falls to approximately 2.5 tesla in the rest of the channel. The solenoid fringe field seen in the region of the dipole magnet varies from 300 to 2000 gauss (near the endplate).The field clamp of the dipole magnet should exclude part of this field. The radial field component, $\mathrm{B}_{\mathrm{r}}$, is given in the figure for a radius of 5 and $10 \mathrm{~cm} . B_{r}$ is present in regions where $B_{z}$ is changing. There is a significant $\mathrm{B}_{\mathrm{r}} \approx 5000$ gauss at 10 $\mathrm{cm}$ localized to the solenoid endplate. There is also some radial feld present for the transition to the higher field near the $\mathrm{H}_{2}$ absorber.

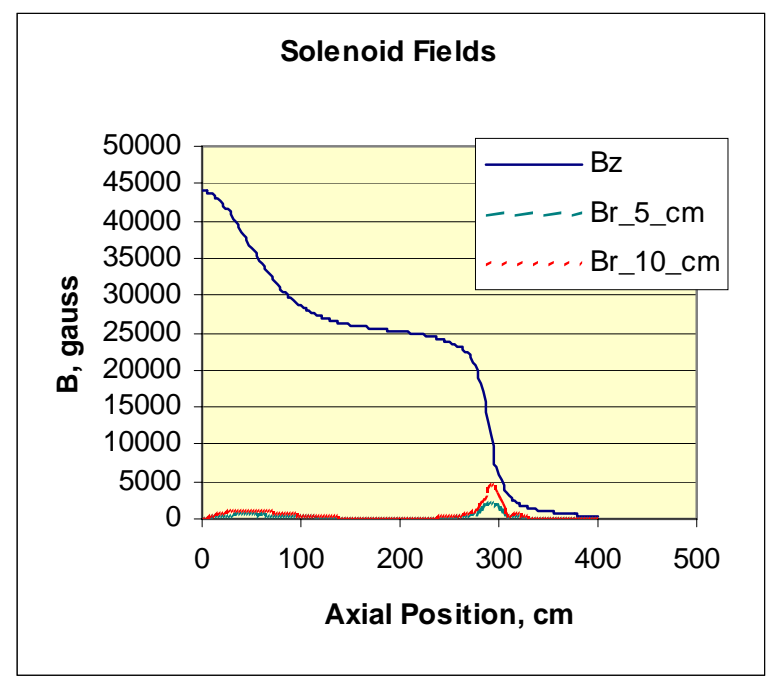

Figure 5: Fields inside long solenoid channel. $B_{z}$ is shown along the solenoid axis. $\mathrm{B}_{\mathrm{r}}$ is shown at a radial position of 5 and $10 \mathrm{~cm}$ off axis.

\section{FIELD FLIP SOLENOID CHANNELS}

In the four corners of the cooling ring between the two $45^{\circ}$ dipole magnets is a short field flip solenoid channel. The coil on each half is $68.43 \mathrm{~cm}$ long and is divided into a high current part $30.82 \mathrm{~cm}$ long near the center carrying $252 \mathrm{amp} / \mathrm{mm}^{2}$ and a low current part $37.61 \mathrm{~cm}$ long carrying $101 \mathrm{amps} / \mathrm{mm}^{2}$. The coils on each half carry current of the opposite sign to produce a field flip in the center. The surrounding iron flux return is designed to contain the field in the region of the solenoid magnet. Figure $6 a$ and $b$ show $B_{Z}$ and $B_{r}$ inside the solenoid at several radial distances. The field is about 1 tesla at the end of the solenoid and falls to 400 gauss in $40 \mathrm{~cm}$, which would be inside the dipole magnet.
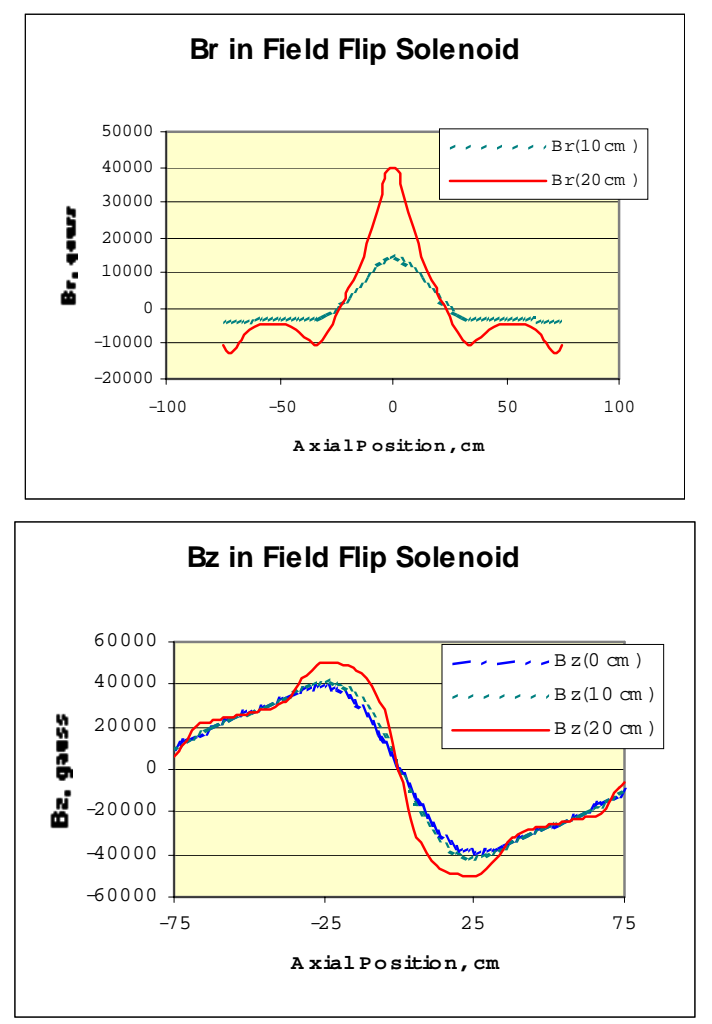

Figure 6: a) $\mathrm{B}_{\mathrm{z}}$ at $\mathrm{r}=0,10$, and $20 \mathrm{~cm}$ inside short solenoid channel. b) $B_{r}$ at $r=10$ and $20 \mathrm{~cm}$.

\section{ACKNOWLEDGEMENT}

This work was supported by the US DOE under Contract Nos. DE-AC02-98CH10886 and DE-AC02-76CH03000.

\section{REFERENCES}

[1] V. Balbekov, Ring Cooler Update, Muon Collaboration Note 189.

[2] TOSCA is part of the Vector Fields Opera3D suite of electromagnetic computation programs. Opera $3 D$ User's Manual, VF-04-00-D2.

[3] Opera2D is a 2D electromagnetic finite element program. Opera2D User's Guide, VF-04-00-A3. 